\title{
Decompositions for the Kakwani poverty index
}

\author{
Oihana Aristondo ${ }^{1}$ Mariateresa Ciommi ${ }^{2}$ \\ ${ }^{1}$ BRIDGE Reserch Group, Departamento de Matemática Aplicada, Universidad del País Vasco, Spain * \\ ${ }^{2}$ Department of Economics and Social Sciences, Università Politecnica delle Marche, Italy ${ }^{\dagger}$
}

\begin{abstract}
Since Sen's seminal article in 1976, it is very known that every poverty measure should be sensitive to the three components of poverty: incidence, intensity and inequality. The paper concentrates on the poverty measure proposed by Kakwani. If the Kakwani index is normalized, an ordered weighted averaging (OWA) operator is obtained. The dual decomposition of the OWA operator into the self-dual core and anti-self-dual remainder allows us to propose a decomposition for this poverty index. Moreover, the inequality term obtained will measure the income inequality and gap inequality of the poor equally.
\end{abstract}

Keywords: Unidimensional Poverty Measurement, Kakwani index, inequality among the poor, Aggregation functions, OWA operators, Dual decomposition

\section{Introduction}

All along, the reduction of poverty has been one of the main purpose of government. To be able to evaluate the efficacy of a poverty-reducing policy, we need a metric that allows both poverty computation and the analysis of its evolution over time. Sen [20] argues that any poverty measurement consists in solving two distinct problems: the identification of the poor in the society and the aggregation of the information about poverty in a summary statistics. The identification of the poor is done by considering an income threshold, the so-called poverty line. The individuals below the poverty line are considered as poor and the others as non poor. For Sen [20] the aggregation step essentially consists on choosing an appropriate poverty measure that combines what Jenkins and Lambert [13] call the three ' $I$ ' of poverty: incidence, intensity and inequality among the poor. In conclusion, any poverty measure should be a function of the number of poor people in the society, the incidence, to the extent of the shortfall of the poor, the intensity, and should take into account the inequality among the poor.

Since the seminal work of Sen [20], a great number of poverty measures accounting for the three ' $I$ ' has

\footnotetext{
*oihana.aristondo@ehu.es

$\dagger$ †email: m.ciommi@univpm.it

Authors gratefully acknowledge the funding support of Departamento de Educación, Política Lingüistica y Cultura del Gobierno Vasco under the project IT568-13. O. Aristondo gratefully acknowledges also the funding support of the Spanish Ministerio de Educación y Ciencia under the project ECO2012-31346, cofunded by FEDER.
}

been introduced in the literature. ${ }^{1}$ In addition, different poverty decompositions have been proposed for the same poverty index. In particular, the inequality among the poor component could refer to the inequality of the income of the poor or to the inequality of the gap of the poor. In the measurement of inequality, the Pigou-Dalton principle plays a crucial role. The axiom requires that a transfer of income from a poor individual to a richer one entails an increase in the inequality among the society. This axiom could be interpreted as the counterpart of the Sen's [20] Transfer axiom which demands that a regressive transfer of income, a transfer from a poorer to a richer, between poor individuals must increase the level of poverty. However, a regressive transfer of income could be also interpreted as a regressive transfer of gap. That is, a transfer of income from a richer to a poorer on incomes entails a transfer of gap from the poorer on incomes to the richer on gaps. In addition, if we focus on shortfalls the richer on incomes is now the poorer on gaps and the poorer on incomes is the now richer on gaps. As a consequence, a poverty measure should increase independently to how the inequality component involved in the decomposition is defined, in terms of incomes or shortfalls. However, the choice between income and shortfall inequality is not innocuous. Moreover, different choices between income and gap may lead to contradictory results.

A similar problem arises in other economic fields in which bounded variables are involved. In recent years, several scholars tried to impose properties to ensure robust measurement. For instance, Lambert and Zheng [17] introduced a consistence property ensuring that achievement and shortfall inequality ranking should not be reversed. In the other hand, Erregers [7] proposes a strongest form, defining perfect complementary indicators as those indicators that measure income inequality and shortfall inequality equally. Finally, Lambert and Zheng [17] and Lasso de la Vega and Aristondo [18], [2] propose a unified framework in which income and shortfall distributions can be jointly analyzed.

Following this literature, we propose two alternative decompositions for the Kakwani poverty index [16] in terms of the three components of poverty. Firstly, we review the existing decomposition of Kakwani index in terms of the inequality of incomes. Then, we propose a new decomposition in terms of

\footnotetext{
${ }^{1}$ See Chakravarty [6], Foster, Greer and Thorbecke [9] and Shorrocks [21] among others.
} 
the inequality of gaps. Unfortunately, we obtain contradictory results for inequality of incomes and gaps. To avoid the possible misunderstandings, besides these two decompositions, we propose an additional decomposition for the Kakwani poverty index, where the third component related to the inequality among the poor is a perfect complementary index. We will reach this new decomposition using ordered weighted averaging operators, hereafter OWA operators, as in Garcia-Lapresta et al. [11] and Aristondo et al. [1]. We prove that the Kakwani poverty index could be interpreted as an OWA operator. By consequence, we can apply the dual decomposition of aggregation function into dual core and anti-self dual remainder proposed by Garcia-Lapresta and Marques Pereira [10]. In particular, we show that the dual core and anti-self dual remainder can be reinterpreted as a measure of the intensity of poverty and the inequality among the poor, respectively. In addition, the anti-self duality of the remainder will force the remainder to be a perfect complementary indicator, that is, an inequality measure that measures equally the inequality of income and the inequality of gap. These poverty components will allow policy makers to determine the increase or decrease of poverty and the sources of this changes in a consistent way.

The rest of the paper is organized as follows. Section 2 presents basic notations for the poverty measurement. A definition of the Kakwani poverty index as well as its preliminary decompositions completes section 3. A description of the OWA operator and its properties are provided in Section 4. The section also includes the definition of the self dual core and the anti-self dual remainder. Our proposal for the decomposition of the Kakwani poverty index based on incidence, intensity and inequality concludes the section. In Section 5, an empirical application for some European countries using EU-SILC data for some years is carry out. Finally, Section 6 is devoted to concluding remarks.

\section{Notations and definitions}

In what follows, we introduce basic notations and definitions used in poverty measurement. Points in $[0, \infty)^{n}$ are denoted $\boldsymbol{x}=\left(x_{1}, \ldots, x_{n}\right)$, with $\mathbf{1}=(1, \ldots, 1), \mathbf{0}=(0, \ldots, 0)$ and for every $x \in$ $[0, \infty), x \cdot \mathbf{1}=(x, \ldots, x)$. Given $\boldsymbol{x}, \boldsymbol{y} \in[0, \infty)^{n}$, $\boldsymbol{x} \geq \boldsymbol{y}$ means $x_{i} \geq y_{i}$ for every $i \in\{1, \ldots, n\}$, and $\boldsymbol{x}>\boldsymbol{y}$ means $\boldsymbol{x} \geq \boldsymbol{y}$ and $\boldsymbol{x} \neq \boldsymbol{y}$. So doing, $\boldsymbol{x}$ represents the income distribution vector of a population of $n \geq 2$ individuals such that $x_{i}$ stands for the income of $i$-th individual. The set of income distributions is $D=\bigcup_{n \in \mathbb{N}}[0, \infty)^{n}$. For a given $\boldsymbol{x} \in D$, let $x_{(1)} \leq \cdots \leq x_{(n)}$ and $x_{[1]} \geq \cdots \geq x_{[n]}$ denote the non decreasing and nonincreasing rearrangement of the coordinates of $\boldsymbol{x}$, respectively. In particular, $x_{(1)}=\min _{i}\left\{x_{i}\right\}=x_{[n]}$ and $x_{(n)}=\max _{i}\left\{x_{i}\right\}=x_{[1]}$. A permutation $\sigma$ on $\{1, \ldots, n\}$ is denoted as $\boldsymbol{x}_{\sigma}=\left(x_{\sigma(1)}, \ldots, x_{\sigma(n)}\right)$ and the arithmetic mean as $\mu(\boldsymbol{x})=\left(x_{1}+\cdots+x_{n}\right) / n$.

As defined by Sen [20], the analysis of poverty involves two steps: the identification of the poor and the aggregation of their individual poverty levels into a composite poverty measure. The identification requires the choice of a poverty line $z \in$ $(0, \infty)$ that establishes a cut-off point for poor and non poor. An individual $i$ is identified as poor if $x_{i}<z$ and as non poor if $x_{i} \geq z .^{2}$ We denote by $q=q(\boldsymbol{x}, z)$ the number of the poor people in the society. For a distribution $\boldsymbol{x}$, we define the poor distribution and its mean as, $\boldsymbol{x}_{q}=\left(x_{(1)}, \ldots, x_{(q)}\right)$ and $\mu\left(\boldsymbol{x}_{q}\right)=\left(x_{(1)}+\cdots+x_{(q)}\right) / q$, respectively. The second step, the aggregation, assigns a numerical value to each distribution that determines the overall level of poverty. That is, a poverty measure is a non-constant function $P: D \times[0, \infty) \rightarrow \mathbb{R}$ whose value $P(\boldsymbol{x}, z)$ denotes the degree of intensity of the poverty associated with an income distribution $\boldsymbol{x}$ and the poverty line $z$.

\section{Poverty measures}

The headcount ratio is the first poverty measure introduced in the literature. It is defined as the ratio between the number of poor people $q$ and the population size $n$

$$
H=H(\boldsymbol{x}, z)=\frac{q}{n} .
$$

It ranges from zero to one, nobody is poor and everybody is poor, respectively. This poverty measure satisfies focus, replication invariance and symmetry axioms. ${ }^{3}$ However, it violates both monotonicity and transfer axioms. ${ }^{4}$ In conclusion, this measure does not capture neither the intensity of the poverty nor the inequality among the poor.

With the intention of measuring the intensity of the poverty the normalized poverty gaps are introduced. For incomes that are below the poverty line, the normalized gap is the relative distance between the income value and the poverty line and for incomes above, it is zero. Formally:

$$
g_{i}=\max \left\{\frac{z-x_{i}}{z}, 0\right\} .
$$

We denote the censored normalized income gap vector as $\boldsymbol{g}=\left(g_{1}, \ldots, g_{n}\right)$ and the normalized gap vector of the poor as $\boldsymbol{g}_{q}=\left(g_{1}, \ldots, g_{q}\right)$. If we compute

\footnotetext{
${ }^{2}$ Donaldson and Weymark [4] define two different ways to identify the poor: the weak and the strong definition. In particular, we use the weak form.

${ }^{3}$ Focus axiom demands independence of the index from the non-poor people. Replication invariance axiom claims that replications of the distribution do not change the index value and the symmetry axiom entails that the name and the position do not matter.

${ }^{4}$ Monotonicity axiom requires an increase in poverty with a reduction in the income level of a poor individual. Transfer axiom states that a poverty measure decreases with a (progressive) transfer of income from a poor to another poorer individual.
} 
the mean of the normalized gap vector of the poor, we obtain another very extensively used measure of poverty, the so-called Income gap ratio, $M$, and defined as:

$$
M=M(\boldsymbol{x}, z)=\mu\left(\boldsymbol{g}_{q}\right)=\frac{1}{q} \sum_{i=1}^{q} g_{[i]}=1-\frac{\mu\left(\boldsymbol{x}_{q}\right)}{z}
$$

where $g_{[1]} \geq \cdots \geq g_{[q]}$ and $x_{(1)} \leq \cdots \leq x_{(q)}<z$. However, this index does not reflect the inequality among the poor. In addition, even if the poverty gap measure satisfies monotonicity axiom, it violates transfer axiom. Therefore, this measure does not take into account the inequality component.

To avoid the lack of sensitivity to inequality among the poor, Sen [20] proposes a new poverty measure. The particularity of this measure is that it places greater weights for poorer individuals.

Definition 1 The Sen Poverty index $S:[0, \infty)^{n} \times$ $(0, \infty) \rightarrow[0,1]$ is defined as follow

$$
S(\boldsymbol{x}, z)=\frac{2}{(q+1) n} \sum_{i=1}^{q}(q+1-i) g_{[i]}
$$

Sen also proves that the poverty measure (1) may be also formulated in terms of the normalized poverty gap $M(\boldsymbol{x}, z)$, the Headcount ratio $H(\boldsymbol{x}, z)$ and the Gini measure of inequality $G(\cdot)$ as follows:

$$
\left.S(\boldsymbol{x}, z)=H\left[M+\frac{q}{q+1}(1-M) G\left(\boldsymbol{x}_{p}\right)\right)\right]
$$

or alternatively:

$$
S(\boldsymbol{x}, z)=H(\boldsymbol{x}, z) M(\boldsymbol{x}, z)\left[1+\frac{q}{q+1} G\left(\boldsymbol{g}_{p}\right)\right]
$$

where $G\left(\boldsymbol{x}_{p}\right)$ and $G\left(\boldsymbol{g}_{p}\right)$ are the Gini coefficient of the distribution of incomes and gaps, respectively. Equation (2) and (3) show that Sen index can be written in terms of the three components of poverty where the inequality components are measured in terms of incomes or gaps, respectively.

However, Kakwani [16] retains that a poverty measure should be also sensitive to the absolute rank of the individuals involved in any income transfer. In other words, given a fixed difference of amount of income between any two poor individuals and a fixed amount to transfer, then the effect on the poverty index of the transfer must be larger to lower is the income of the pair involved. This property is the main motivation for the formulation of the Kakwani's parametric family also known as Kakwani poverty index.

Definition 2 (Kakwani poverty index) The Kakwani Poverty index $K_{k}:[0, \infty)^{n} \times(0, \infty) \rightarrow[0,1]$ is defined as follow, for any $k \geq 0$

$$
K_{k}(\boldsymbol{x}, z)=\frac{q}{n \varphi_{q}(k)} \sum_{i=1}^{q}(q+1-i)^{k} g_{[i]}
$$

where $k$ is the poverty aversion and $\varphi_{q}(k)=\sum_{i=1}^{q} i^{k}$.
Originally, the Kakwani Index was formulated by Nanok C. Kakwani [14] to measure the progressivity of tax systems. It ranges from -2 to 1 . A negative value occurs if the financing source is regressive; a positive one if it is progressive, and a value equal to zero states a proportional financing source. In this case it represents the difference between the concentration index of health care payments and the Gini index of health care payments and the Gini index of income distribution. In addition, it found several applications such as the study of equity in health care expenditures. ${ }^{5}$

Since $K_{k}(\boldsymbol{x}, z)$ depends also on $k$ that represents the number of people among the poor with income high at least as $i$, usually literature refers to it as the Kakwani's parametric family. ${ }^{6}$ For $k=0$ it reduces to the so-called Poverty gap ratio, defined as the product between the Headcount ratio ,$H(\boldsymbol{x}, z)$, and the Income gap ratio, $M(\boldsymbol{x}, z)$, as: $K_{0}(\boldsymbol{x}, z)=H(\boldsymbol{x}, z) M(\boldsymbol{x}, z)$. This measure only reflects the incidence and the intensity of poverty and it is insensitive to the inequality among the poor. For $k=1$, Kakwani's index reduces to the Sen poverty index (1), that is: $K_{1}(\boldsymbol{x}, z)=S(\boldsymbol{x}, z)$

In literature, there is an additional formulation for the Kakwani's parametric family that involves the Headcount ratio, the Income gap ratio and a measure of inequality.

Proposition 1 The Kakwani's parametric family can be decomposed as follows

$$
K_{k}(\boldsymbol{x}, z)=H\left(M+(1-M) E_{k}\left(\boldsymbol{x}_{p}\right)\right)
$$

where $E_{k}\left(\boldsymbol{x}_{p}\right) \in[0,1]$ is an inequality measure of the income of the poor:

$$
E_{k}\left(\boldsymbol{x}_{p}\right)=\frac{1}{\mu\left(\boldsymbol{x}_{p}\right) \phi_{q}(k)} \sum_{i=1}^{q}\left(\mu\left(\boldsymbol{x}_{p}\right)-x_{(i)}\right)(q+1-i)^{k}
$$

and $x_{(1)} \leq \cdots \leq x_{(q)}<z$ are the poor incomes in the distribution $\boldsymbol{x}$.

\section{Proof 1 The proof is provided in Kakwani [16].}

This inequality measure satisfies Pigou dalton principle, normalization, symmetry and replication invariance axioms. ${ }^{7}$ Following this result, we propose an alternative decomposition for Kakwani index in terms of the inequality of the gap of the poor.

\footnotetext{
${ }^{5}$ See Kakwani et al. [15] and Van Doorslaer et al. [22] among others.

${ }^{6}$ Parameter $k$ may be interpreted as an intensity coefficient.

${ }^{7}$ Pigou-Dalton principle axiom requires that a transfer of income from a poor individual to a richer one entails an increase of the inequality. Normalization axiom requires that the inequality is equal to zero if all the individuals have the same income. Symmetry and Replication invariance axioms require that inequality does not change by a permutation and a replication, respectively.
} 
Proposition 2 Kakwani index can be rewritten as follows:

$$
K_{k}(\boldsymbol{x}, z)=H M\left(1+E_{k_{g}}\left(\boldsymbol{g}_{p}\right)\right)
$$

where $E_{k_{q}}\left(\boldsymbol{g}_{p}\right) \in[0,1]$ is an inequality measure of the gap of the poor:

$$
E_{k_{g}}\left(\boldsymbol{g}_{p}\right)=\frac{1}{\mu\left(\boldsymbol{g}_{p}\right) \phi_{q}(k)} \sum_{i=1}^{q}\left(g_{(i)}-\mu\left(\boldsymbol{g}_{p}\right)\right) i^{k}
$$

and $g_{(1)} \leq \cdots \leq g_{(q)}$ are the poor gaps in the distribution $\boldsymbol{g}$.

Proof 2 To show that the decomposition holds, we rewrite $K_{k}(\boldsymbol{x}, z)$ in terms of the gap:

$$
K_{k}(\boldsymbol{x}, z)=\frac{q}{n \phi_{q}(k)} \sum_{i=1}^{q}(q+1-i)^{k} g_{[i]}
$$

Using both definition of $H$ and $M$ and some manipulations, we get:

$$
=H M \sum_{i=1}^{q} \frac{(q+1-i)^{k}}{M \phi_{q}(k)}\left(g_{[i]}-M+M\right)=
$$

Using $\sum_{i=1}^{q}(q+1-i)^{k}=\sum_{i=1}^{q}(i)^{k}=\phi_{q}(k) w e$ obtain:

$$
\begin{gathered}
=H M\left(1+\sum_{i=1}^{q} \frac{(i)^{k}}{M \phi_{q}(k)}\left(g_{(i)}-M\right)\right)= \\
=H M\left(1+E_{k_{g}}\left(\boldsymbol{g}_{p}\right)\right)
\end{gathered}
$$

that completes the first part of the proof.

To prove $E_{k_{g}}\left(\boldsymbol{g}_{p}\right)$ is an inequality measure satisfying Pigou dalton principle, normalization, symmetry and replication invariance axioms is straightforward.

This inequality measure also satisfies the Pigou dalton principle, normalization, symmetry and replication invariance axioms.

Remark $1 \quad E_{k}\left(\boldsymbol{x}_{p}\right)$ and $E_{k_{g}}\left(\boldsymbol{g}_{p}\right)$ are related by the following relationship:

$$
M(\boldsymbol{x}, z) E_{k}\left(\boldsymbol{x}_{p}\right)=(1-M(\boldsymbol{x}, z)) E_{k_{g}}\left(\boldsymbol{g}_{p}\right)
$$

However, as already mentioned, the choice between the inequality index of the poor incomes and the poor gaps is not innocuous. The following example may better illustrate this problem.

Example 1 Let $\boldsymbol{x}^{1}=(2,10,20,35)$ and $\boldsymbol{x}^{2}=$ $(3,20,23,37)$ be two income distributions and $z=$ 40 an hypothetic poverty line. We compute the corresponding poverty gap distributions $\boldsymbol{g}^{1}=$ $\left(\frac{38}{40}, \frac{30}{40}, \frac{20}{40}, \frac{5}{40}\right)$ and $\boldsymbol{g}^{2}=\left(\frac{37}{40}, \frac{20}{40}, \frac{17}{40}, \frac{3}{40}\right)$. The $E_{k}$ index of the income distributions concludes that the inequality among the poor is higher in the former than in the latter for $k=1$ and $k=2, E_{1}\left(\boldsymbol{x}^{1}\right)=0.325>$ $0.253=E_{1}\left(\boldsymbol{x}^{2}\right)$ and $E_{2}\left(\boldsymbol{x}^{1}\right)=0.528>0.427=$ $E_{2}\left(\boldsymbol{x}^{2}\right)$. Nevertheless, this conclusion is reversed if the $E_{k g}$ index of the gap distributions is computed since $E_{1 g}\left(\boldsymbol{g}^{1}\right)=0.234<0.273=E_{1 g}\left(\boldsymbol{g}^{2}\right)$ and $E_{2 g}\left(\boldsymbol{g}^{1}\right)=0.381<0.460=E_{2 g}\left(\boldsymbol{g}^{2}\right)$.
In the following section, we propose an alternative decomposition of the Kakwani index, based on OWA operators, that overcomes the lack of consistency shown before.

\section{An alternative decomposition using OWA operators}

\subsection{Definitions}

We summarize basic notations on aggregation functions, OWA operators and their decompositions into the self-dual core and the anti-self-dual remainder.

We restrict the domain to $[0,1]^{n}$. We begin by defining standard properties of real functions defined on $[0,1]^{n}$. $^{8}$

Definition 3 Let $A:[0,1]^{n} \longrightarrow R$ be a function.

1. A is symmetric if $A\left(\boldsymbol{x}_{\sigma}\right)=A(\boldsymbol{x})$, for any permutation $\sigma$ on $\{1, \ldots, n\}$ and all $\boldsymbol{x} \in[0,1]^{n}$.

2. $A$ is monotonic if $\boldsymbol{x} \geq \boldsymbol{y} \Rightarrow A(\boldsymbol{x}) \geq A(\boldsymbol{y})$, for all $\boldsymbol{x}, \boldsymbol{y} \in[0,1]^{n}$. Moreover, $A$ is strictly monotonic if $\boldsymbol{x}>\boldsymbol{y} \Rightarrow A(\boldsymbol{x})>A(\boldsymbol{y})$, for all $\boldsymbol{x}, \boldsymbol{y} \in[0,1]^{n}$.

3. $A$ is invariant for translations if $A(\boldsymbol{x}+t \cdot \mathbf{1})=$ $A(\boldsymbol{x})$, for all $t \in \mathbb{R}$ and $\boldsymbol{x} \in[0,1]^{n}$ such that $\boldsymbol{x}+t \cdot \mathbf{1} \in[0,1]^{n}$. On the other hand, $A$ is stable for translations if $A(\boldsymbol{x}+t \cdot \mathbf{1})=A(\boldsymbol{x})+t$, for all $t \in \mathbb{R}$ and $\boldsymbol{x} \in[0,1]^{n}$ such that $\boldsymbol{x}+t \cdot \mathbf{1} \in[0,1]^{n}$

4. $A$ is invariant for dilations if $A(\lambda \cdot \boldsymbol{x})=A(\boldsymbol{x})$, for all $\lambda>0$ and $\boldsymbol{x} \in[0,1]^{n}$ such that $\lambda$. $\boldsymbol{x} \in[0,1]^{n}$. On the other hand, $A$ is stable for dilations if $A(\lambda \cdot \boldsymbol{x})=\lambda A(\boldsymbol{x})$, for all $\lambda>0$ and $\boldsymbol{x} \in[0,1]^{n}$ such that $\lambda \cdot \boldsymbol{x} \in[0,1]^{n}$.

5. $A$ is idempotent if $A(x \cdot \mathbf{1})=x$, for all $x \in$ $[0,1]$.

6. $A$ is compensative if $x_{(1)} \leq A(\boldsymbol{x}) \leq x_{(n)}$, for all $\boldsymbol{x} \in[0,1]^{n}$.

7. $A$ is self-dual if $A(\mathbf{1}-\boldsymbol{x})=1-A(\boldsymbol{x})$, for all $\boldsymbol{x} \in[0,1]^{n}$

8. $A$ is anti-self-dual if $A(\mathbf{1}-\boldsymbol{x})=A(\boldsymbol{x})$, for all $\boldsymbol{x} \in[0,1]^{n}$.

9. $A$ is S-convex if $A(\boldsymbol{y})<A(\boldsymbol{x})$, for all $x, y \in$ $[0,1]$ where $\boldsymbol{y}$ is obtained from $\boldsymbol{x}$ by a progressive transfer.

Definition 4 Let $\left\{A^{(k)}\right\}_{k \in \mathbb{N}}$ be a sequence of functions, with $A^{(k)}:[0,1]^{k} \longrightarrow \mathbb{R}$ and $A^{(1)}(x)=x$ for every $x \in[0,1]$. We say that $\left\{A^{(k)}\right\}_{k \in \mathbb{N}}$ is invariant for replications if it holds that

$$
A^{(m n)}(\overbrace{\boldsymbol{x}, \ldots, \boldsymbol{x}}^{m})=A^{(n)}(\boldsymbol{x})
$$

for all $\boldsymbol{x} \in[0,1]^{n}$ and any number of replications $m \geq 2$ of $\boldsymbol{x}$.

Definition 5 A function $A:[0,1]^{n} \longrightarrow[0,1]$ is called an $n$-ary aggregation function if it is monotonic and $A(\mathbf{0})=0, A(\mathbf{1})=1$. An aggregation

\footnotetext{
${ }^{8}$ See Fodor and Roubens [8], Calvo et al. [5], Beliakov et al. [3], Grabisch et al. [12] and García-Lapresta and Marques Pereira [10] for more details.
} 
function is said to be strict if it is strictly monotonic. ${ }^{9}$

Following García-Lapresta and Marques Pereira [10] we know that every aggregation function is compensative. However, in general, aggregation functions don't satisfy neither self-duality nor anti-selfduality properties. Nevertheless, self-duality, antiself-duality and stability for translations are important properties of aggregation functions and will play an important role in this paper.

\subsection{Dual decomposition and OWA operators}

Any aggregation function $A$ can be decomposed as the sum of a self-dual function and an anti-self-dual function, defined as follows:

Definition 6 Let $A:[0,1]^{n} \longrightarrow[0,1]$ be an aggregation function. The functions $\widehat{A}, \widetilde{A}:[0,1]^{n} \longrightarrow[0,1]$ define as

$$
\begin{aligned}
& \widehat{A}(\boldsymbol{x})=\frac{A(\boldsymbol{x})-A(\boldsymbol{1}-\boldsymbol{x})+1}{2} \\
& \widetilde{A}(\boldsymbol{x})=\frac{A(\boldsymbol{x})+A(\boldsymbol{1}-\boldsymbol{x})-1}{2}
\end{aligned}
$$

are called the core and the remainder of the aggregation function $A$, respectively.

$\widehat{A}$ is self-dual and it is called the self-dual core of the aggregation function $A$. Note that $\widehat{A}$ is also an aggregation function since $\widehat{A}(\mathbf{0})=0, \widehat{A}(\mathbf{1})=1$ and $A$ satisfies monotonicity. On the other hand, $\widetilde{A}$ is called the anti-self-dual remainder of the aggregation function $A$ and it satisfies the anti-self-duality property. However, it is not an aggregation function since $\widetilde{A}(\mathbf{0})=\widetilde{A}(\mathbf{1})=0$. It is easy to prove that $-0.5 \leq \widetilde{A}(\mathbf{x}) \leq 0.5$ for every $\mathbf{x} \in[0,1]^{n} .^{10}$

Remark 2 Every aggregation function can be decomposed as the sum of the self-dual core, $\widehat{A}$, and the anti-self-dual remainder, $\widetilde{A}$, that is $A(\mathbf{x})=$ $\widehat{A}(\mathbf{x})+\widetilde{A}(\mathbf{x})$.

The self-dual core $\widehat{A}$ is also an aggregation function and inherits from the aggregation function $A$ the properties of continuity, idempotency, compensativeness, symmetry, strict monotonicity, stability for translations, and invariance for replications, whenever $A$ has these properties.

On the other hand, the anti-self-dual remainder $\widetilde{A}$ is not an aggregation function. In this case, the selfdual core $\widetilde{A}$ inherits from the aggregation function $A$ the properties of continuity, symmetry, invariance for replications, and also strict $S$-convexity, whenever $A$ has these properties.

\footnotetext{
${ }^{9}$ For simplicity, the $n$-arity is omitted whenever it is clear from the context.

${ }^{10}$ For more information see García-Lapresta and Marques Pereira [10].
}

Yager [23] introduces special aggregation functions similar to weighted means called ordered weighted averaging (OWA) operators.

Definition 7 Given a weighting vector $\boldsymbol{w}=$ $\left(w_{1}, \ldots, w_{n}\right) \in[0,1]^{n}$ satisfying $\sum_{i=1}^{n} w_{i}=1$, the OWA operator associated with $\boldsymbol{w}$ is the aggregation function $A_{w}:[0,1]^{n} \longrightarrow[0,1]$ defined as follows,

$$
A_{w}(\boldsymbol{x})=\sum_{i=1}^{n} w_{i} x_{[i]}
$$

where $x_{[1]} \geq \cdots \geq x_{[n]}$ as usual in the literature on OWA operators. In addition, if the weights are (strictly) non increasing, $w_{1} \geq \cdots \geq w_{n}$, then the OWA operator $A_{w}$ will be (strictly) $\bar{S}$-convex.

Remark 3 García-Lapresta and Marques Pereira [10] show that the self-dual core and the anti-selfdual remainder of an OWA operator can be defined in the same way as follows:

$$
\begin{aligned}
& \widehat{A}_{w}(\boldsymbol{x})=\sum_{i=1}^{n} \frac{w_{i}+w_{n-i+1}}{2} x_{[i]} \\
& \widetilde{A}_{w}(\boldsymbol{x})=\sum_{i=1}^{n} \frac{w_{i}-w_{n-i+1}}{2} x_{[i]}
\end{aligned}
$$

In particular, The self-dual core $\widehat{A}_{w}$ is an $O W A$ operator since $\sum_{i=1}^{n}\left(w_{i}+w_{n-i+1}\right) / 2=1$. However, the anti-self-dual remainder $\widetilde{A}_{w}$ is not an $O W A$ operator since $\widetilde{A}_{w}$ is not an aggregation function and $\sum_{i=1}^{n}\left(w_{i}-w_{n-i+1}\right) / 2=0$.

\subsection{A decomposition of Kakwani index}

To introduce an alternative decomposition of Kakwani index using OWA operators, we need to rewrite the Kakwani index as follows:

$$
K_{k}(\boldsymbol{x}, z)=H \sum_{i=1}^{q} \frac{(q+1-i)^{k}}{\phi_{q}(k)} g_{[i]}=H A_{k}\left(\boldsymbol{g}_{p}\right)
$$

The following proposition proves that the term multiplying the Headcount ratio, $A_{k}\left(\boldsymbol{g}_{p}\right)$, corresponds to an OWA operator that satisfies several axioms.

Proposition 3 The function $A_{k}:[0,1]^{q} \longrightarrow[0,1]$ applied to the normalized poverty gaps,

$$
A_{k}\left(\boldsymbol{g}_{p}\right)=\sum_{i=1}^{q} w_{i} g_{[i]}, \quad w_{i}=\frac{(q+1-i)^{k}}{\phi_{q}(k)}
$$

is an OWA operator. In addition, it satisfies continuity, idempotency (hence, compensativeness), symmetry, strict monotonicity, stability for translations and strict $S$-convexity. 
Proof 3 Firstly, since $K$ is a continuous function $A_{k}$ also satisfies continuity. Secondly, since $\sum_{i=1}^{q} w_{i}=\sum_{i=1}^{q} \frac{(q+1-i)^{k}}{\phi_{q}(k)}=1, A_{k}$ is idempotent, hence compensative, and stable for translations. Then, the positivity of the weights implies strict monotonicity. Finally, as we have $w_{1}>\cdots>w_{q}$, then the strict decrese of the weights implies the strict $S$-convexity of the function $A_{k}$.

Using the definition of the self-dual core and the anti-self-dual reminder of $A_{k}$ we propose an additional decomposition of the Kakwani poverty index in terms of the three components of poverty. The following proposition formalizes our intuition.

Proposition 4 The Kakwani poverty index can be decomposed as follows:

$$
\begin{aligned}
K_{k}(\boldsymbol{x}, z) & =H(\boldsymbol{x}, z) A_{k}\left(\boldsymbol{g}_{p}\right) \\
& =H(\boldsymbol{x}, z)\left(\widehat{A}_{k}\left(\boldsymbol{g}_{p}\right)+\widetilde{A}_{k}\left(\boldsymbol{g}_{p}\right)\right)
\end{aligned}
$$

where $\widehat{A}_{k}\left(\boldsymbol{g}_{p}\right)$ and $\widetilde{A}_{k}\left(\boldsymbol{g}_{p}\right)$ are the self-dual core and the anti-self-dual reminder of $A_{k}\left(\boldsymbol{g}_{p}\right)$.

$$
\begin{aligned}
& \widehat{A}_{k}\left(\boldsymbol{g}_{p}\right)=\sum_{i=1}^{q} \frac{(q+1-i)^{k}+i^{k}}{2 \phi_{q}(k)} g_{[i]} \\
& \widetilde{A}_{k}\left(\boldsymbol{g}_{p}\right)=\sum_{i=1}^{q} \frac{(q+1-i)^{k}-i^{k}}{2 \phi_{q}(k)} g_{[i]}
\end{aligned}
$$

Proof 4 Since $w_{i}=(q+1-i)^{k} / \phi_{q}(k)$ and $w_{q-i+1}=i^{k} / \phi_{q}(k)$, we obtain

$$
\begin{aligned}
\widehat{A}_{k}\left(\boldsymbol{g}_{p}\right) & =\sum_{i=1}^{q} \frac{w_{i}+w_{q-i+1}}{2} g_{[i]} \\
& =\sum_{i=1}^{q} \frac{(q+1-i)^{k}+i^{k}}{2 \phi_{q}(k)} g_{[i]} \\
\widetilde{A}_{k}\left(\boldsymbol{g}_{p}\right) & =\sum_{i=1}^{q} \frac{w_{i}-w_{q-i+1}}{2} g_{[i]} \\
& =\sum_{i=1}^{q} \frac{(q+1-i)^{k}-i^{k}}{2 \phi_{q}(k)} g_{[i]} .
\end{aligned}
$$

Note that for $k=1$ the self-dual core of $A_{1}$ is the mean of the normalized poverty gap vector, $\widehat{A}_{1}\left(\boldsymbol{g}_{p}\right)=\mu\left(\boldsymbol{g}_{p}\right)$ and the anti-self-dual reminder of $A_{1}$ is the absolute Gini index of the normalized poverty gap vector multiplied by $q /(q+1)$, that is $\widetilde{A}_{1}\left(\boldsymbol{g}_{p}\right)=\frac{q}{q+1} G_{A}\left(\boldsymbol{g}_{p}\right)$.

Remark 4 By Proposition 3 and Remark 2, $A_{k}$ is idempotent, symmetric, strictly monotonic and stable for translations. As mentioned before, the self-dual core $\widehat{A}_{k}$ inherits the properties of idempotency, symmetry, strict monotonicity and stability for translations from the OWA operator $A_{k}$. The strictly monotonicity implies that $\widehat{A}_{k}$ is increasing in the gap of a poor person. The stability for translations means that equal absolute changes in all poor gaps lead to the same absolute change in $\widehat{A}_{k}$. However, $\widehat{A}_{k}$ is not $S$-convex, and then it goes against the Pigou-Dalton transfer principle. Consequently, these properties can be regarded as basic properties of a poverty intensity index.

In turn, the remainder $\widetilde{A}_{k}$ is symmetric, fulfils $\widetilde{A}_{k}\left(g_{1}, \ldots, g_{q}\right)=0$ if and only if $g_{1}=\cdots=g_{q}$. From Proposition $3 \widetilde{A}_{k}$ is also strictly $S$-convex, and consequently the anti-self-dual reminder $\widehat{A}_{k}$ satisfy the Pigou-Dalton transfer principle. Therefore, $\widetilde{A}_{k}$ can be interpreted as a measure of inequality among the poor individuals.

Hence, Proposition 4 shows that Kakwani index can be decomposed into the three components of poverty. The Headcount ratio $H$ is a measure of the incidence of poverty. The intensity of poverty as the poverty depths of poor individuals in the society is summarized by the $\widehat{A}_{k}$. Finally, the inequality of the poor gap distribution is captured by the dispersion measure $\widetilde{A}_{k}$, which provides the sensitivity to the inequality among the poor. As mention, the inequality among the poor refers to the inequality of the poor gap distribution. The following proposition shows that the inequality component $\widetilde{A}_{k}$ is consistent with respect to incomes and gaps, since it measures equally the inequality of incomes and gaps.

Proposition 5 For the inequality measure $\widetilde{A}_{k}$, the following equivalence holds:

$$
\widetilde{A}_{k}\left(\boldsymbol{g}_{p}\right)=\widetilde{A}_{k}\left(\boldsymbol{x}_{p} / z\right)
$$

Proof 5 Since $\widetilde{A}_{\widetilde{A}_{k}}$ is an anti-self-dual function it satisfies $\widetilde{A}_{k}(\boldsymbol{x})=\widetilde{A}_{k}(\mathbf{1}-\boldsymbol{x})$, then $\widetilde{A}_{k}\left(\boldsymbol{g}_{p}\right)=\widetilde{A}_{k}(\mathbf{1}-$ $\left.\boldsymbol{g}_{p}\right)=\widetilde{A}_{k}\left(\boldsymbol{x}_{p} / z\right)$

As above mentioned, the main result of proposition 5 is that the inequality component of the Kakwani index measures income and gap inequality equally.

Last proposition shows the invariance properties that are satisfied by the inequality measure $\widehat{A}_{k}$ defined before.

Proposition 6 The inequality measure $\widetilde{A}_{k}$ is invariant for translations and stable for dilations.

\section{Proof 6 The proof is straightforward.}

That is, the $\widetilde{A}_{k}$ component remains invariant if the gaps of all the poor individuals are increased by the same amount. Hence, $\widetilde{A}_{k}$ can be considered an absolute inequality measure.

\section{Empirical findings}

In this section, we illustrate the different decompositions for the Kakwani poverty index proposed in previous sections, using European Union Survey on 
Table 1: Poverty Decompositions

\begin{tabular}{l|c|c|ccc|ccc}
\hline & $\mathrm{z}$ & $\% H$ & $K_{1}$ & $K_{2}$ & $K_{3}$ & $\widehat{A}_{1}$ & $\widehat{A}_{2}$ & $\widehat{A}_{3}$ \\
\hline Germany 2005 & 15410.5 & $16.69 \%$ & 0.0628 & 0.0739 & 0.0817 & 0.2649 & 0.2758 & 0.2868 \\
Germany 2009 & 17018.8 & $18.46 \%$ & 0.0756 & 0.0883 & 0.0970 & 0.2917 & 0.3014 & 0.3111 \\
\hline Hungary 2005 & 1573.9 & $13.30 \%$ & 0.0441 & 0.0523 & 0.0579 & 0.2314 & 0.2423 & 0.2531 \\
Hungary 2009 & 3004.6 & $11.13 \%$ & 0.0333 & 0.0398 & 0.0445 & 0.2060 & 0.2178 & 0.2295 \\
\hline Latvia 2005 & 926.1 & $22.50 \%$ & 0.0956 & 0.1122 & 0.1238 & 0.3009 & 0.3129 & 0.3249 \\
Latvia 2009 & 4249.5 & $26.84 \%$ & 0.1246 & 0.1421 & 0.1539 & 0.3451 & 0.3507 & 0.3564 \\
\hline Lithuania 2005 & 954.9 & $22.31 \%$ & 0.0998 & 0.1163 & 0.1276 & 0.3209 & 0.3316 & 0.3423 \\
Lithuania 2009 & 2342.2 & $21.80 \%$ & 0.0909 & 0.1067 & 0.1179 & 0.2966 & 0.3087 & 0.3208 \\
\hline
\end{tabular}

Income and Living Conditions (EU-SILC) data. We compare 26 countries in two different years: 2005 and 2009. The unit of analysis are the individuals. By consequence, the household disposable income computed for individuals is used as variable of interest. ${ }^{11}$ Finally, we fix the poverty line $z$ to the 60 percent of the median national equivalent household income in each year. ${ }^{12}$ Using this definition of the poverty line, a person is considered poor if he lives in a household who income is below this threshold. To allow cross-section comparisons for the two periods, namely 2005 and 2009, all the monetary values are converted into Purchasing Power Standards (PPS), in order to account the differences in the purchasing power of different national currencies. ${ }^{13}$ Of course, this only affects on the mean income of the poor people and the poverty line, since all the poverty and inequality indices are scale invariant.

Table 1 and Table 2 show the results for 2005 and 2009 for four EU countries, namely Germany, Hungary, Latvia and Lithuania. We have chosen these countries because we think that they present controversial results and they are of particular interest to show our contributions. Table 1 shows the incidence, intensity and poverty results between 2005 and 2009 for Germany, Hungary, Latvia and Lithuania. The first two columns list the poverty line $z$ and the Headcount ratio,columns 3, 4 and 5 show the poverty measures of Kakwani for $k=1$, $k=2$ and $k=3$. In addition, the last three columns report the intensity components for the proposed three decompositions. On the other hand, in Table 2 we can observe the corresponding inequality components for every inequality decompositions.

If we focus on Table 1 and we look at Germany and Latvia, we have higher values on poverty in 2009 than 2005 for the computed three Kakwani poverty measures. If we focus on the three poverty components, we can also observe higher levels of incidence and intensity in 2009. With respect to the inequality

\footnotetext{
${ }^{11}$ To move from the household level to the individual ones, we use as equivalence scale the square root of the number of individual in each household.

${ }^{12}$ We observe that fixing the poverty line as a function of the distribution is not innocuous. In fact, this implies the violation of the Focus Axiom. However, this is the usual way to determine the poverty line when real data are involved.

${ }^{13}$ Including those countries that share a common currency, for example the Euro.
}

components, Germany displays more poor inequality on incomes in 2009 but less poor inequality on gaps. However, the consistent indices introduced in this paper allow us to conclude that the inequality of the poor has increased in this period for Germany. On the other hand, Latvia shows lowest values in gap inequality for 2009 but nothing can be concluded for the inequality of incomes, contradictory results are obtained for the three inequality components. Also in this case, if we focus on the three corresponding consistent inequality components, inequality decreases from 2005 to 2009 for any inequality measure both if we focus on incomes and on gaps. Now, if we have a look to Hungary and Lithuania, the Headcount ratio and the three Kakwani poverty measure decrease in this period. However, analyzing Hungary's and Lithuania's poor inequality in the two years, opposite results are reached when comparing the poor inequality respect to incomes and gaps. In particular, we obtain lower values for all poverty indices in 2009 than in 2005. We conclude that the inequality of the income distribution of the poor falls for Hungary and Lithuania. However, the trend is reversed if we switch to gaps as the variable of interest. Also in this last case, looking at the consistent indices, we can affirm that inequality among the poor decreases in Hungary and Lithuania between 2005 and 2009 .

\section{Concluding remarks}

The recent literature on poverty measurement stresses the importance for a index to account for Intensity, Incidence and Inequality. In this paper, we propose alternative decompositions for the Kakwani Poverty Index in terms of the above mentioned components. We have shown that an order weighted averaging operator is underlying in the definition of the Kakwani index. The properties inherited in the proposed decomposition allow us to obtain an inequality component that measures income inequality of the poor and gap inequality of the poor equally. The illustration using EU-SILC data for 2005 and 2009 for 26 European countries shows the gripping ability of our decomposition. Since the decomposition we propose allows for consistent inequality component for the inequality among the poors, we believe that it could be a good instrument for policy 
Table 2: Inequality Components

\begin{tabular}{l|ccc|ccc|ccc}
\hline & $E_{1}\left(\mathbf{x}_{p}\right)$ & $E_{2}\left(\mathbf{x}_{p}\right)$ & $E_{3}\left(\mathbf{x}_{p}\right)$ & $E_{1 g}\left(\mathbf{g}_{p}\right)$ & $E_{2 g}\left(\mathbf{g}_{p}\right)$ & $E_{2 g}\left(\mathbf{g}_{p}\right)$ & $\widetilde{A}_{1}\left(\mathbf{g}_{p}\right)$ & $\widetilde{A}_{2}\left(\mathbf{g}_{p}\right)$ & $\widetilde{A}_{3}\left(\mathbf{g}_{p}\right)$ \\
\hline Germany 2005 & 0.1514 & 0.2420 & 0.3051 & 0.4202 & 0.6717 & 0.8469 & 0.1113 & 0.1667 & 0.2024 \\
Germany 2009 & 0.1667 & 0.2639 & 0.3304 & 0.4050 & 0.6409 & 0.8024 & 0.1181 & 0.1772 & 0.2145 \\
\hline Hungary 2005 & 0.1306 & 0.2100 & 0.2656 & 0.4337 & 0.6973 & 0.8820 & 0.1004 & 0.1506 & 0.1825 \\
Hungary 2009 & 0.1178 & 0.1916 & 0.2443 & 0.4541 & 0.7385 & 0.9416 & 0.0935 & 0.1403 & 0.1704 \\
\hline Latvia 2005 & 0.1771 & 0.2828 & 0.3566 & 0.4114 & 0.6569 & 0.8285 & 0.1238 & 0.1857 & 0.2253 \\
Latvia 2009 & 0.1821 & 0.2818 & 0.3485 & 0.3457 & 0.5349 & 0.6615 & 0.1193 & 0.1789 & 0.2169 \\
\hline Lithuania 2005 & 0.1861 & 0.2949 & 0.3696 & 0.3938 & 0.6240 & 0.7821 & 0.1264 & 0.1896 & 0.2296 \\
Lithuania 2009 & 0.1713 & 0.2741 & 0.3469 & 0.4062 & 0.6502 & 0.8227 & 0.1205 & 0.1807 & 0.2197 \\
\hline
\end{tabular}

makers.

\section{References}

[1] O. Aristondo, J. L. García-Lapresta, C. Lasso de la Vega and R. A. Marques Pereira, The Gini index, the dual decomposition of aggregation functions, and the consistent measurement of inequality, International Journal of Intelligent Systems, 27, 132-152,2012.

[2] O. Aristondo and C. Lasso de la Vega, Measuring the inequality of bounded distributions: a joint analysis of attainments and shortfalls, Research on Economic Inequality, 21, 33-52,2013.

[3] G. Beliakov, A. Pradera and T. Calvo, Aggregation Functions: A Guide for Practitioners, Heidelberg: Springer, 2007.

[4] D. Donaldson and J. A. Weymark, Properties of Fixed-Population Poverty Indices, International Economic Review, 27(3), 667-88, 1986.

[5] T. Calvo, A. Kolesárova, M. Komorníková and R. Mesiar, In: Calvo T, Mayor G, Mesiar R, editors. Aggregation Operators: New Trends and Applications. Heidelberg, Germany: Physica-Verlag; 2002, pp. 3-104.

[6] S. R. Chakravarty, A new index of poverty, Mathematical Social Sciences, vol. 6(3), 307-313, 1983.

[7] G. Erreygers, Can a single indicator measure both attainment and shortfall inequality? $J$. Health Econ 28, 885-893, 2008.

[8] J. Fodor, M. Roubens, Fuzzy, Preference Modelling and Multicriteria Decision Support. Dordrecht, The Netherlands: Kluwer Academic Publishers, 1994.

[9] J. Foster, J. Greer and E. Thorbecke, A class of decomposable poverty measures, Econometrica, 52(3): 761-765, 1984.

[10] J. L. García-Lapresta and R. A. Marques Pereira, The self-dual core and the anti-self-dual remainder of an aggregation operator. Fuzzy Sets and Systems, 159, 47-62, 2008.

[11] J. L. Garcia-Lapresta, C. Lasso de laVega, R. A. Marques Pereira, A. M. Urrutia, A class of poverty measures induced by the dual decomposition of aggregation functions, Int $J$ Uncertainty Fuzziness Knowl-Based Syst, 18, 493-511, 2010.

[12] M. Grabisch, J. L. Marichal, R. Mesiar, E. Pap, Aggregation Functions. Cambridge: Cambridge
University Press, 2009.

[13] S. Jenkins and P. Lambert, Three 'I' of poverty curves and poverty dominance: TIPs for poverty analysis, Research on Economic Inequality, 8, JAI Press, Greenwich, 39-56, 1998.

[14] N.C. Kakwani, Measurement of Tax Progressivity: An International Comparison, Economic Journal, 87(345), 71-80, 1977.

[15] N.C. Kakwani, A. Eagstaff and E. van Doorslaer, Socioeconomic inequalities in health: measurement, computation, and statistical inference, Journal of Econometrics, 77(1), 87-103, 1997.

[16] N.C. Kakwani, On a Class of Poverty Measures, Econometrica, 48, 437-446, 1980.

[17] P. Lambert and B. Zheng, On the consistent measurement of achievement and shortfall inequality, Journal of Health Economics, 30, 214219, 2011.

[18] C. Lasso de la Vega and O. Aristondo, Proposing indicators to measure achievement and shortfall inequality consistently, Journal of Health Economics, 31(4), 578-583,2012.

[19] A.K. Sen, Poverty: an ordinal approach to measurement, Econometrica, 44(2), 219-221, 1976.

[20] A.K. Sen, Development As Freedom, Oxford: Oxford University Press, 1999.

[21] A.F. Shorrocks, Revisiting the Sen poverty index.Econometrica, 63, 1225-1230, 1995.

[22] E. Van Doorslaer, A. Wagstaff, H. Bleichrodt, S. Calonge, U. G. Gerdtham, M. Gerfin, ... and O. Winkelhake, Income-related inequalities in health: some international comparisons. Journal of health economics, 16(1), 93-112, 1997.

[23] R. R. Yager, Ordered weighted averaging operators in multicriteria decision making. IEEE Transactions on Systems, Man and Cybernetics, 8: 183-190, 1988. 